\title{
ASPECTOS DE LA ONOMÁSTICA DE RONDA
}

\author{
Manuel Álvarez García \\ Manuel Ariza Viguera \\ Josefa Mendoza Abreu
}

\section{INTRODUCCIÓN}

Reconquistada Ronda por los Reyes Católicos en el año 1485 se procedió, como era habitual, al repartimiento de sus bienes (tierras y casas, fundamentalmente), entre las diferentes personas que fueron a repoblarla. El único requisito que la Corona exigía para obtener la posesión de los bienes adjudicados era la permanencia hasta cinco años en la ciudad. El incumplimiento de esta norma conllevaba la pérdida de lo percibido y consiguientemente el paso a un nuevo propietario De ahí los reajustes de propiedad que se observan a lo largo del Repartimiento. A ello habría que añadir que por estas fechas se produce una epidemia de peste que lleva a la muerte de algunos de estos primitivos propietarios, cuyos bienes también serán nuevamente distribuidos. Todo esto alarga considerablemente la lista de los repobladores que aparecen en el Libro de Repartimiento, de cuya nominación nos vamos a ocupar. La gran riqueza de matices permite un estudio más amplio. En este primer acercamiento vamos a tratar fundamentalmente los siguientes aspectos:

1) Estructura general de la nominación.

2) El topónimo utilizado como apellido o nombre de familia.

3) La nominación indirecta

4) La nominación femenina.

\section{ESTRUCTURA DE LA DENOMINACIÓN}

La estructura más general que caracteriza la denominación de los habitantes de Ronda es la constituida por nombre + apellido. Los nombres más frecuentes son Juan, Alfonso, Diego, Fernando, Francisco, Pedro, etc.; los menos usuales Alberto, Benjamín, Blas, Gabriel, Lázaro, Matías y Sebastián, que corresponden cada uno de ellos a una sola persona. Y, como era de esperar, dado su uso entre personas de origen árabe o hebreo, una sola persona se llama Alazraque («escribano del arábigo», p.242), Alí (p.85) o Israel. En 
este sentido una orden real deja claramente explícita la imposibilidad de que los judíos puedan vivir en la ciudad de Ronda tras su reconquista:

Ansy mismo es nuestra merled e voluntad que en la dicha gibdad de Ronda non pueda hivir ni morar judío nin estar en ella de tres días arriba, eçebto Ysrraél nuestro tramújar [trujaman] de arávigo. (p.92)

En cuanto a los apellidos pueden tener diversa procedencia. Generalmente se trata de un antropónimo: «Gonçalo Fernandes» (198), «Fernando Gonzales» (347), o topónimo: «Alfonso de Andújar» (440), «Antonio de Sahagund» (359). Y en menor proporción proceden de un oficio: «Diego Pescador» (162), «Juan Sillero» (197, 394,...), o de una característica peculiar: «Diego (el) Tuerto» (1 3 7, 34 1), «Juan el Loco» (175); sin que se observen diferencias sociales ni en lo que se refiere a los nombres ni en los apellidos, al menos en los dos primeros tipos citados, pues lo mismo encontramos un «Juan Dávila», «regidor» $y$ «juez» $(169,170, \ldots)$ que otro de oficio «barvero» (396); o «Juan Fernandes», «chançiller» $(308,366)$ y otro «pastor» $(313)$. Lo cual es lógico teniendo en cuenta la época, que permite, como es sabido, que, por ejemplo, un padre se llame «Pero Diaz de Verona» (363) y el hijo «Diego Martin» (363).

Ahora bien, además de esa estructura general aparecen diversas variantes, condicionadas en un buen número de casos por una serie de factores entre los que podemos destacar los siguientes:

(a) La necesidad de distinción de las personas, sobre todo cuando hay coincidencia formal de nombres, por lo que a veces aparece un segundo apellido, o alguna característica especifica (oficio, etc.).

(b) El prestigio social y consiguientemente el grado de conocimiento que se tiene de cada persona, lo que lleva a una doble posibilidad: 1) que al individuo tenga que nombrársele también por referencias a otras personas «más significativas»; 2) el caso opuesto, es decir, que su nombre o apellido, unido a veces a su profesión, sea suficiente para determinarlo.

(c) El conocimiento que los diferentes medidores y repartidores tienen de cada una de las personas. Este generalmente suele ser bueno, pero a veces lleva a que una misma persona aparezca citada de dos formas distintas, como veremos, o a que su nombre sea totalmente desconocido para unos pero no para otros (Vid. 3).

Probablemente por esas razones con bastante frecuencia tras la denominación, a veces antes, aparece la profesión u oficio de la persona citada: «Bartolomé Sanchez, carniceros (174), «Pero Hernandes, cavallerizo» (428), «Juan Garçia, vallestero» (364), «el calero Juan Martin». Rasgo que suele ser distinguidor cuando hay coincidencia de nombre y apellido: «Bartolomé Sanchez, carnicero (174), «Bartolomé Sanchez, lobero» (379), «Bartolomé Sanchez, tendero» (171), «Bartolomé Sanchez, zapatero» (173). También es bastante usual que en vez de la profesión, o además, encontremos el lugar de procedencia con el mismo valor distinguidor (Vid 2). Siguiendo con el mismo nombre, encontrarnos: «Bartolomé Sanchez de Villarreal» (154), «Bartolomé Sánchez de Lebrija» (178). Tanto el 
nombre de la profesión como, y sobre todo, el topónimo, que generalmente se incorpora como segundo apellido, pueden pasar a sustituir al primero o quedar como único apellido: «Alfonso Garcia de Cordova» (429) es nombrado también como «Alfonso de Córdova, albannir» (429), «Alfonso Garçia Cavallero» $(310,378)$ como «Alfonso Cavallero», quizás por seguir esa estructura general de nombre + apellido.

Por otro lado nos encontramos con que en lugar del nombre + apellido aparece sólo el nombre o sólo el apellido seguido o precedido de otros rasgos identificadores como oficio, parentesco, etc. Veamos las variantes:

Rasgo identificador + nombre: «Maestre Miguel(I)» (289, 298, 195), «Maestre Sancho» (149, 191, 345), «Maestre Ramiro» (148), que por un documento real sabemos que era artillero $(109,185)$. En algunas ocasiones se precisa colocando también el oficio; así: «Maestre Al(f)onso» $(168,373)$ aparece también «como Maestre Alonso, fisico», e incluso con más precisión «Maestre Alonso, fysico, escudero de las guardas» (341). 0 bien puede registrarse el nombre completo, es decir nombre y apellido; así, «Don Sancho», que es la denominación general $(261,249,285,458,461)$, en dos ocasiones lo encontramos como «don Sancho de Rojas» $(134,136)$ (es el único que sistemáticamente lleva este tratamiento en todo el Repartimiento). «Mosén Fernando» $(94,343)$ aparece también como «Fernando Rejón» (136, 177, 279), «Mosén Fernando Rejón» (288) o «Mosén Fernando de Rejón» (345).

Nombre + rasgo identificador: Con bastante frecuencia cuando aparece sólo el nombre, éste suele ir seguido del oficio, si es suficientemente significativo: «Innigo, jurado» (174, $212,235,237,359, \ldots$ ) (una vez «Enigo», p.134), "Juanote, artillero" (346, 408). Aunque en este caso el nombre solo suele ser más frecuente $(346,461,136,288, \ldots$ ); y en dos ocasiones aparece con otro sinónimo como: «Juanote, lombardero» $(273,157)$ pero sólo por otros documentos reales conocemos su apellido «Juanote de Ostaes» (109).

También con el nombre solo, o seguido del oficio en menor proporción encontrarnos el nombre de Enrique (157), (sin posibilidad de confusión pues es el único que aparece y además por las tierras que recibe) que antes figuraba corno «Enrique Xastre» (191), en otras ocasiones como «maestre Enrrique Sastre» y en otras como «Enrrique, jubetero» (372) o «Enrrique Jubetero» (302). En éste, como en otros casos, es dificil saber si el oficio ha pasado a integrar el apellido o no; aunque la alternancia «Sastre» - «Jubetero» parece indicar que aún no.

En otros casos lo identificador, más que el oficio, es la persona con quien trabajan (Vid. también 3); así lo vemos en «Pedro, azemilero de Fuenseca» $(170,585)$, «Pedro, criado de Juan Darze» (199, 765), «Alonso, el criado de Juan Dávila» (177), «Fernando, criado de Juan Dávila» $(175,615)$.

$\mathrm{Y}$ aunque aparecen también con apellido, el rasgo identificador, es decir, el nombre de la persona para quien trabajan se mantiene siempre: «Alonso Garçia, criado de Juan Dávila», «Fernando de Paredes, criado de Juan Dávila el regidor» $(146,425)$, donde, como vemos, se añade incluso el oficio del «amo». 
Un caso significativo es el que vemos en el siguiente texto: «A la mano derecha otra casa que tiene Antón, criado de Miguel Martín, herrero; es desposado e la compró de ... ; queda al dicho Antón Herrero por vezindad... Diése mas al dicho Antón Herrero, la tienda ...» $(182,655)$.

Y desde luego no faltan los ejemplos de identificación por parentesco, cuando el pariente es el más conocido por su oficio o prestigio social: «Alberto, fijo de Miguell Martín, herrero» (412), «Alonso, hijo de Juan de Lara, jurado» (302), «Fernando, yerno de Juan de Annora» (291), o por tratarse de una menor o mujer (Vid. 3): «Mariça, hija de Juan de Segovia que finó» (190).

En algunas ocasiones el hijo puede aparecer con el apellido del padre, con referencia también a éste o sin ella: «Alberto Martín, fijo de Miguel Martín, herrero» (391). «Françisco, su hijo» [de Juan Fernandes de Sanhelizes] (141) aparece más tarde como: «Francisco, fijo de Santhelizes», y posteriormente como: «Françisco de Santhelizes» (447). Y lo mismo ocurre con «Lorenço, hijo de Juan Garcia de Badajos» (340), que pasa a denominarse «Lorenço de Badajos», con el segundo apellido y topónimo del padre, igual que en el caso anterior: «Lorenço, hijo de Juan Garçia de Badajos, que fallesció; es a Penna Serrada e queda allí al dicho Lorenço un terçio" (340), "Lorenço de Badajos tiene asentado un terçio de cavallería de tierra por vezyndad; midióse su partido en Penna Serrada; quédale allí proveydo" (388).

Un caso especial lo encontramos cuando es el nombre sólo el que sirve para identificar: "Pedro de la Torre, padre de Julio" (383). Este Julio parece ser la misma persona que antes aparece como "Julián" sin más (144) y después como "Julian de la Torre" (441) (Vid. Indice del autor).

Los mismos esquemas que acabamos de ver aparecen también cuando el elemento general de denominación es el apellido, aunque en este caso y con mucha mayor frecuencia éste suele aparecer solo.

Rasgo identificador + apellido: "el alcayde Zurita" (351), "el bachiller Padilla" (135), "el jurado Bustamante" (329), “el jurado Rabín” (176), "el alcayde Cerrato” (426), "el sennor Fonseca" (276), que después lo encontramos como el "sennor Antonio de Fonseca e Alarcón" (276) (en los documentos reales como "Antonio de Fonseca" $(96,110$.... ) y una vez como "Antonio de Alarcón e de Fonseca" (126), pero que generalmente es nombrado Fonseca. Como puede verse, cuando el rasgo precede al nombre, se trata siempre de cargos públicos, y por tanto conocidos. Sin embargo, si va pospuesto, no hay limitaciones, ya se trate de un oficio o cualquier otra particularidad.

Apellido + rasgo identificador: "Monterroso, cabtivo" (398), "Cespedes, tintorero" (161), "Monesterio, abad" (332), "Contreras, arriero" (425) "Cifontes, secretario del comendador mayor" (311), "Castillo, el azemilero" (177, 401), "Castillo, el regidor" (332).

En los casos en que para la denominación se recurre, además del apellido, a la afinidad con otras personas, es curioso observar cómo la relación e incluso el nombre de esas personas puede no aparecer explícito, sino a través de lo que llamarnos nominación 
indirecta (Vid. §3): "Moreno, criado de Horosco" (152, 163, 387,...), "Aranda, criado del licenciado" (177), "Aranda, el del licenciado" (411). Pero, como decíamos, no son menos frecuentes las veces en que la nominación se hace a través del apellido exclusivamente, ya sea porque el nombre completo ha aparecido alguna vez, y en cualquier caso porque así la persona es suficientemente conocida. Así lo vemos, por ejemplo, en el ya citado Fonseca, en Cifuentes, etc.: "Fuenseca, siete araçadas" (172), "Cifuentes, vendió a Juan Castillo" (172) (así, o sin diptongación, Cifontes, se repite ocho veces). El nombre de este último no aparece nunca, lo más que conocemos es que era "secretario del comendador" (31 l).

Pero además, aparece el apellido sólo en casos en que por coincidencias se puede prestar a confusión, como podría ocurrir con Horosco que aparece identificado como medidor $(434,438 \ldots$ partidor $(169,177, \ldots)$ y texedor $(169,430)$, y sin elemento diferenciador alguno, Horosco $(299,308$...). Indudablemente debe tratarse de dos personas, una, de oficio texedor, la otra, uno de los medidores del Repartimiento y como tal suficientemente conocido como para denominársele sólo con el apellido: Horosco.

También puede aparecer el apellido solo porque ése sea el único elemento conocido; generalmente porque esa persona ha muerto o ya no vive en la población: "Otra casa junto con ésta que hera de Ahumada, e se fue antes del tienpo e la vendio" (182), "seys olivos que se quitaron a Arnedo, que se fue e vendyo sin residir" (432), "fue dada a Brios, que Dios aya" (159), "los diez olivos que se avian dado a Rangel, que se fue" (436). Y en esta misma línea hay que considerar los casos en que la nominación se hace a través del nombre del oficio, que puede integrarse como apellido: "Pero Sanchez Cantador, diósele por vezindad una cuarta de vinna" (309)., "Pero Cantador tiene cinco olivos" (450), "entre Gonçalo Ximenes y el Cantador" (293,- 294), o quedar como tal nombre de oficio y apodo; es lo que ocurre, por ejemplo, en: "El Bonetero, media cuarta" (169), "diósele media cuarta de vinna que hera del Bonetero, que se fue sin resydir" (310), y sólo al final del Repartimiento conocemos su nombre: "dyéronsele nueve olivos de Gonçalo Sanches, bonetero, que se fue" (404).

\section{EL TOPÓNIMO UTILIZADO COMO APELLIDO O NOMBRE DE FAMILIA}

Es muy frecuente el uso de topónimos formando parte de las nominaciones en el Libro de Repartimiento de Ronda, hecho que, por otra parte, tiene raíces muy antiguas. Las estructuras de nominación en las que puede aparecer un apellido toponomástico son diversas en este texto:

(a) Nombre de pila + adjetivo gentilicio: "Martin Gallego" (1 56).

(b) Nombre de pila + topónimo: "Alonso de Coynbra" (1 4 1), "Alvaro de Çalamea" (342). En algún caso este topónimo parece utilizarse como elemento para distinguir a un individuo de otro: "Juan de Cuenca" (161) "Juan de Lora" (399), "Pedro de Çamora" (170) , "Pedro de Consuegra" (288).

(c) Nombre de pila + topónimo + término indicador de oficio o profesión que se utilizó en esta ocasión como diferenciador, al ser coincidentes los dos anteriores: "Alonso de Córdova, albannir" (141), «Alonso de Córdova, 
ballestero» (169), «Alonso de Córdova, mercader» (292). Puede aparecer intercalado un apodo entre el topónimo y el vocablo indicador de oficio: «Alonso de Córdova, el Cojo, escudero» (95).

(d) Nombre de pila + topónimo + topónimo: el segundo topónimo puede actuar aquí como diferenciador entre individuos: «Juan del Castillo» (157) / «Juan del Castillo de Teba» (408).

(e) Nombre de pila + apellido + topónimo donde este último elemento se emplea como distinguidor: «Alfonso García de Villagarçía» (248), «Alfonso García de Salamanca» (363), «Alfonso García de Usagre» (427), «Alfonso Garcia de Córdova» (429), «Alfonso García de Toro» (437), «Alfonso García de Villasanta» (445)

(f) Aparece, por último, un tipo de nominación, de la que forma parte un topónimo, donde a veces funciona como elemento distinguidor el topónimo y en otros casos el indicador de oficio o los dos a la vez, con evidente redundancia a efectos de la diferenciación de individuos: «Alfonso López, armero» (194), «Alfonso López de Santo Espíritu, labrador» (284), «Alfonso López de Luque» (395), «Alfonso López, hortelano» (428), «Alfonso López de Siruela, cabrero» (428), «Alfonso López de Guadalupe» (432).

Queremos, finalmente, hacer referencia al origen de los repobladores teniendo en cuenta el empleo que se hace de los topónimos corno apellidos. Los más frecuentes corresponden a lugares de la Andalucía Occidental (Sevilla, Utrera, Écija, Córdoba, Montilla, Fuenteovejuna, Jerez, Vejer, Zalamea, Aroche, etc.), de Badajoz (Badajoz, Llerena, Monesterio, Usagre, Azuaga, etc.) y de Jaén (Jaén, Baeza). Fuera de esto es de notar la presencia de Zamora (cuatro individuos), Toro (también cuatro), Toledo (seis), Avila (dos), Arévalo (tres), Galicia (cuatro), Valencia (tres) y una larga lista de topónimos que aparecen citados como nombres de familia en uno o dos individuos y que se refieren a localidades de toda la geografía peninsular. Como muestra de ello, he aquí algunos ejemplos: Segovia (dos), Madrid (dos), León (uno), Logroño (uno), Oviedo (uno), Tineo (uno), Salamanca (uno), Cuenca (uno), Coimbra (uno).

\section{LA NOMINACIÓN INDIRECTA}

En este tercer apartado vamos a detenernos en el estudio de dos aspectos particulares de la nominación en el Libro del Repartimiento de Ronda: la que, en principio, llamaremos «nominación indirecta» y la femenina. Son dos aspectos interesantes puesto que constituyen excepciones en la tendencia general de nominación. Claro es, como se trata de un reparto de posesiones, lo que cuenta, en principio, es la unidad familiar, por lo que las mujeres de los pobladores y sus hijos pequeños no necesitan nominación particular; pero -como veremos- la mujer no la suele llevar incluso si es protagonista del repartimiento. Bien es cierto que la innominación es obligada cuando se trata de persona indefinida como en: «el fisico que la cibdad toviere» (202), «el bachiller de la gramática que subçeda de uno en otro que en la çibdad oviere» (198), o cuando el nombre se ignora por tratarse de alguien que a) ya no habita en la ciudad y del que sólo se tiene el recuerdo: «un aperador de Trevinno que se fue» (158), «el barbero de Teba, que se fue» (160 y 303), «uno que no es 
vezino» (301), «un barbero moro» (242); b) ha fallecido: «el odrero que fallescio» (170 y 311), o cuando ha sido imposible saber su nombre: «otro que no supe su nombre» (148).

No falta algún caso dudoso, como «el que se fue por sahumerio para la pestilencia» (290), que bien podría ser el que vemos más adelante: "e un olivo en el partido de Fernán Lopes de Ocanna, el que fue por sahumeryo para la pestilencia" (300).

Junto a esto puede existir el caso contrario: que perdure el nombre y no la persona: «Femand Sánchez, que no se sabe quién es» (404), «Bartolomé Rodríguez, que non saben quien es» (436). Pero en otras ocasiones la innominación puede deberse a antonomasia: el dean, el tesorero, el cambiador, el teniente, el notario, el letrado de la ciudad, etc. Si nos fijamos, son todos cargos públicos, generalmente unipersonales, aunque no siempre ocurre así, por lo que a veces, es necesaria una mayor concreción: «el dean de Canaria», «el dean de Çamora», «el alcayde de Gaulin», etc.

En la misma línea deben considerarse «el bachiller dell escuela» $(145,168)$ y «el bachiller de la gramática" $(277,303,304,348)$, sin que pueda pensarse en un uso indefinido, como veíamos antes, puesto que el contexto parece indicar persona concreta: «Pascual e el bachiller de la gramática, dióse a estos dos por merçed otra suerte más adelante desta» (277). Y ello pese que, al parecer, existía otro, seguramente jubilado: «el bachiller de la gramática, el Ciego» (292). Es curioso, además que esta innominación produce una confusión nominal (valga la redundancia): el bachiller de la escuela es llamado «Bernaldo d'Escobar» en la p.292 y «Fernando de Escovar» en la p.412.

A tabú pueden deberse la innominaciones siguientes: «fijos del que aorcaron» (365), «el que degolló a su muger» (236 y 296), aunque líneas adelante nos dice su nombre de pila: «el dicho Francisco, que mató a su muger» (236). Quizá al mismo motivo se deba la innominación plural en «los jurados que mataron» $(166,285,333$, etc.).

A motivación social se debe la innominación que se produce a veces con los criados: «el mayoral de Juan de Avila» (160), «el criado de Lezcano» (162), «un criado de Horozco» (162), «un moço de Laso» (169). Es de notar que sus amos son personajes importantes de la ciudad.

El caso contrario lo tenemos en «el licenciado de la Fuente» $(268,401,402)$, al que incluso en una ocasión se le llama simplemente "el licenciado", y cuyo nombre conocemos por un documento real de 1485 en el que se le nombra regidor perpetuo "El licenciado Juan de la Fuente, nuestro alcalde en la nuestra casa e corte e del nuestro Consejo" (89). Es decir, la misma innominación, en el fondo que veíamos en "el tesorero", que sabemos se llama Ruy López (401), «el cambiador» Aranda y demás cargos.

Junto a lo ya examinado hay casos en donde coexisten la especificación nominal y la referencial: «uno que traxo las bulas, que se llama Pedro de Vaena» (235), «Olivera, el que se casó con la de Bustamante» (374), «Rodrigo Alfonso, que se le fue la muger» (375), o la doble nominal por empleo del apodo: «Fernando de Toledo, que se dezia Sábado malo» (405); con un caso realmente sorprendente: «Vallejo, que se llama Juan Pares» (292). 
Algunos presentan problemas: «un serrano que se dezia Juan Martin de Hinojosa» (161), «un texedor que se dize Juan Martin Sevillano».

\section{LA NOMINACIÓN FEMENINA}

Ya hemos indicado que lo más frecuente es la innominación femenina, o, si se prefiere, la nominación indirecta o referencial: «la muger de Sotomayor» (168), «la suegra de Benito León» (168), «la madre de Montalván» (209), ete. Hay un ejemplo de doble boda: «la muger de Julián de la torre, muger de Pero Ruiz» (441). En «la muger que se casó con ... » (250) se trata de segundas nupcias.

La mujer es llamada también en múltiples ocasiones «la de»: «la de Xaramillo», «la de Sotomayor», «la de Rivas», etc. (300), incluso cuando se vuelve a casar: «Pedro de Toro, su marido de la de Fernando de Aguilar» (234), «Morales, que se casó con la de Pedro del Pino» (184), «Castanneda, casado con la muger de Velasquillo» (184). Sólo en un caso la referencia nominal es el segundo marido: «la muger del jurado Morales, media arançada que fue dada a Brios, que Dios aya, su marido» (159). Claro es que se debe a la importancia social del jurado.

Pero no siempre ocurre así, incluso hay veces en que es el hombre el que tiene como referencia el nombre de su mujer: «el marido de la Mallera» (370), «El Portogués marido de la Castellana» (298). Son estos casos excepcionales, puesto que lo más frecuente es que aparezca también el nombre del marido; es más, la referencia femenina es casi siempre el sobrenombre: «Juan Muñoz, el marido de la Partera» (176), «Alonso Fernández, marido de la Cordovesa» (292). Curiosamente el único ejemplo en que la referencia femenina es onomástica, no se trata del marido sino del yerno: «Diego Martín, yerno de Mari Cana» (391). Sólo hay un ejemplo de doble innominación, masculina y femenina, un personaje llamado «el Platero», sin que sepamos, en principio, si se trata de un apodo o de un oficio; se cita a su mujer y a su hermana: «la muger del Platero» (442), «la hermana del Platero» (133).

Ahora bien, la innominación puede crear confusiones, pues, en otro lugar se cita a «la beata hermana del Platero» (185), de la que en otro lugar se nos dice que se llama «Catalina» (304), ¿es la misma? como veremos no parece, pero por otras referencias del texto. Es decir: una primera duda estaría en saber si «beata» es apodo o profesión; es claro que se trata de una beata medieval, hasta el punto de que en Ronda existió un beaterio; veamos el siguiente párrafo: «A la casa de las beatas diósele media arançada e media quarta de vinna que se dió a Catalina la Beata, hermana del Platero, porque no se le avia de dar por no ser casada» (304). Luego no puede ser la misma de la que se nos dice lo siguiente: «la hermana del Platero, que fynó... esta es muger de Fernán Rodríguez, vallestero del Rey» (160). Curiosamente este personaje tenía también una hermana beata (234), como también tenía una hermana de tal condición «Juan de Lara» (304).

La innominación puede ser también de tipo social, como veíamos en los hombres: «un ama de Juan Dávila» (245), «la criada de Gonçalo Sánchez de la Somoça, que casó con 
Juan Eçija» (411), o por desconocimiento: «una vieja bibda, que vino bibda a la çibdad» (255).

A medio camino entre la innominación y el nombre propio está el apodo; muy frecuente entre las mujeres. Doy la lista: la Bellota, la Calderona, la Candelera vieja, la Carmona, la Castellana, la Cerera, la Cordovesa, la Chacona, la Chapinera, la Galana, la Garlona, la Lechera, la Mallera, la Morena, la Morena d'Archidona, la Partera, la Persevala, la Portuguesa, la Romera vieja, la Sillera, la Tomadiza.

Desconocemos el nombre y el apellido de la inmensa mayoría de las mujeres, como tampoco sabemos cuáles son auténticos apodos o cuáles hacen referencia directa a la profesión. Algunos son feminizaciones de un nombre masculino: la Calderona tiene un hijo que se llama «Juan Calderón» (411), por lo que hay que deducir que su marido se apellidaba de la misma manera; como sucede en este otro ejemplo, mucho más claro: «la de Perseval» (201-2) es llamada también «la Persevala» (201-2).

Finalmente no escasean las nominaciones femeninas. La estructura más frecuente es la de nombre de pila + apellido. Aun en estos casos, es abundante que el nombre vaya acompañado de la referencia «marital»: «Teresa Rodríguez, muger de Miguel Pérez» (349), «Catalina de Luzón, que es casada con Juan Cheryno» (427), «Catalina Fernández, muger que fue de Alvaro de la Cadena» (442), o esta otra estructura mucho más curiosa: «la de Alonso Galán, que se dize Beatriz Sánchez» (192). El nombre de la mujer aparece en gran medida por ser ésta viuda o haber llegado ya viuda a la ciudad; es lo que ocurre con «Marina Gonçález, la Gargona» (215), «Juana Martín de Aroche»(236), «Guiomar Rodríguez» (302), «Mari Fernández, o Hernández la Cavallera» (267, 312, etc. En p.261 se la denomina «la Caballera» sin más).

En algunos ejemplos no tenemos datos concretos, aunque es presumible que se trate de viudas por no nombrarse nunca a sus maridos: «Beatriz Gómez la Tornadiza» (21 1), «Mari o María Cana» (176, 215, 301, 424), «Maria Bivas» (255), «Beatriz Serena» (261), etc. Aunque no es una regla, es más frecuente la denominación onomástica cuando se trata de mujeres casadas o emparentadas con personajes importantes: «Leonor García» (241) está casada con un caballero, «Catalina Bermúdez» (345) está casada con un regidor, «Teresa Rodríguez» (349) está casada con un alcalde, «Catalina de Luzón» (352) debe ser hermana del repartidor, «Mencía de Escalante» (200, etc.) tiene carta real de merced.

Como es conocido, el apellido era de libre elección, sin embargo queremos destacar un caso muy interesante: el de «Elvira Garçía de Aguilera» (364 y 450). Copiamos el texto: «Elvira Garçía de Aguilera, diósele un terçio de cavallería para ella e para sus fijos porque fallesció en esta çibdad su marido Aguilera (... ) sobró en ello el dicho terçio que se da la dicha Aguilera». En donde vemos el uso de la preposición de y el apellido del marido como componente de la denominación de una mujer casada, y, además, el origen de algunas denominaciones con articulo: la Aguilera.

Por último señalaremos que los nombres de pila más frecuentes son Catalina (7 personas), María (5), Marina (4), Beatriz (3), Isabel (2), y, con una Blanca, Elvira, Guiomar, Juana, Leonor, Mencía, Quiteria y Teresa. 
